BI-TP 2010/48

December 2010

\title{
Thermal production of relativistic Majorana neutrinos: Strong enhancement by multiple soft scattering
}

\author{
Alexey Anisimov, Denis Besak 1, Dietrich Bödeker 2 \\ Fakultät für Physik, Universität Bielefeld, D-33615 Bielefeld, Germany
}

\begin{abstract}
The production rate of heavy Majorana neutrinos is relevant for models of thermal leptogenesis in the early Universe. In the high temperature limit the production can proceed via the $1 \leftrightarrow 2$ (inverse) decays which are allowed by the thermal masses. We consider new production mechanisms which are obtained by including additional soft gauge interactions with the plasma. We show that an arbitrary number of such interactions gives leading order contributions, and we sum all of them. The rate turns out to be smooth in the region where the $1 \leftrightarrow 2$ processes are kinematically forbidden. At higher temperature it is enhanced by a factor 3 compared to the $1 \leftrightarrow 2$ rate.
\end{abstract}

\footnotetext{
${ }^{1}$ dbesak@physik.uni-bielefeld.de

${ }^{2}$ bodeker@physik.uni-bielefeld.de
} 


\section{Introduction and motivation}

One of the outstanding problems of standard cosmology is to explain the origin of the asymmetry between matter and antimatter. Without such an asymmetry, all the structures we observe today would have never formed and mankind would not exist. The asymmetry can be expressed as the baryon-to-photon ratio

$$
\frac{n_{B}}{n_{\gamma}}=(6.21 \pm 0.16) \cdot 10^{-10}
$$

whose numerical value is obtained from a combined analysis of data for large-scale structure and the spectrum of the Cosmic Microwave Background [1].

In order to obtain a net baryon asymmetry, only three conditions need to be met, as outlined by Sakharov in his seminal paper [2]. Yet, providing a model that can successfully explain the measured baryon-to-photon ratio remains a challenging task. Several different scenarios how to realize the Sakharov conditions have been devised [3]. In the last decade, leptogenesis [4] has become very popular. The basic idea of most leptogenesis models is to enlarge the particle content of the Standard Model (SM) with heavy Majorana neutrinos. In the simplest realization they interact with the SM particles via a Yukawa coupling to ordinary, left-handed leptons and the Higgs bosons as follows:

$$
\mathscr{L}_{\text {int }}=h_{i j} \bar{N}_{i} \widetilde{\varphi}^{\dagger} \ell_{L j}+\text { h.c. },
$$

where $N$ stands for the Majorana neutrinos, $\ell_{L}$ and $\varphi$ are the left-handed lepton doublet and the Higgs doublet, and $\widetilde{\varphi} \equiv \varepsilon \varphi^{*}$ with $\varepsilon_{\alpha \beta}=-\varepsilon_{\beta \alpha}, \varepsilon_{12}=1$. Finally, the indices $i, j$ label the fermion families, and $h_{i j}$ is the Yukawa coupling matrix which need not be diagonal.

The Majorana neutrinos are unstable and decay both into leptons and antileptons, $N \rightarrow \ell \varphi, N \rightarrow \bar{\ell} \varphi^{\dagger}$. The CP symmetry is violated and the corresponding decay rates are not equal. Therefore, an excess of antileptons over leptons can be generated. The resulting asymmetry is converted into an excess of baryons over antibaryons via the sphaleron transitions which conserve $B-L$ but violate $B+L[5]$. In addition to providing a source for the measured baryon asymmetry, this scenario offers a framework to explain the smallness of the neutrino masses via the seesaw mechanism [6]. This twofold virtue is what makes the scenario of leptogenesis particularly appealing.

Despite a substantial amount of work and progress [7, 8, 9, 10] a complete theory of leptogenesis is still lacking [11]. In this paper we study a type of processes which 
so far has not been considered in this context. We show that they contribute to the production of heavy Majorana neutrinos at leading order in the coupling constants, and we find that their contribution is numerically large. Our results therefore constitute an important step towards a complete treatment of thermal leptogenesis.

We compute the production rate of a Majorana neutrino in a hot electroweak plasma that is fully equilibrated, except for the Majorana neutrinos themselves. The production rate is part of the network of Boltzmann equations that is solved to obtain the baryon asymmetry. Since it sets the initial conditions for leptogenesis, it is also of practical interest to study the production rate by itself. We assume that the number density of Majorana neutrinos is small compared to the equilibrium density, so that the inverse processes which reduce their number density can be neglected. We focus on the lightest Majorana neutrinos $N_{1} \equiv N$, which we assume to be the dominant source of lepton asymmetry.

When the temperature $T$ is sufficiently above the Majorana neutrino mass $M_{N}$, a peculiar type of production mechanism occurs. It was already considered in Refs. [8, 9]. Interactions with the hot plasma generate thermal masses, which are much bigger for SM particles than for the Majorana neutrinos. Therefore the SM particles can become "heavier" than the Majorana neutrino, and the decay of a Higgs boson into Majorana neutrino and SM lepton can become possible. Since thermal masses are parametrically small compared to the typical particle momentum, all momenta involved in this decay are nearly collinear. In this paper we show that there are additional nearly collinear processes, involving soft electroweak gauge interactions, which contribute to the leading order production rate.

We focus on the leading order in the $\mathrm{SU}(2)$ and $\mathrm{U}(1)$ gauge couplings $g$ and $g^{\prime}$, the top quark Yukawa coupling constant $h_{t}$ and the Higgs self-coupling $\lambda$. We do not consider the production via $2 \leftrightarrow 2$ scattering processes 3 . For the power counting we assume that all these couplings are of the same order and collectively refer to them by g. All other SM couplings are neglected. We perform the computation in the hightemperature regime where $M_{N} \ll T$. This allows us to formally treat the mass of the Majorana neutrino as being soft, $M_{N} \sim g T$, and therefore parametrically of the same order as the thermal Higgs and lepton masses. We demonstrate that even at leading order the production cannot simply be understood in terms of scattering processes

\footnotetext{
${ }^{3}$ In the literature one can find several calculations of $2 \leftrightarrow 2$ scattering rates (see, e.g. 8, 12, 13, [14, 15]). However, to the best of our knowledge, there is no calculation which consistently treats all leading order thermal effects.
} 
involving only a handful of particles. ' $N$-strahlung' and inverse decay processes involving multiple interactions mediated by soft electroweak gauge bosons are not suppressed despite the large number of vertices. The emission occurs almost collinearly, so that propagators are nearly on-shell and compensate the suppression. In position space the radiated particle and its source overlap over large distances, and the interference of different interactions cannot be neglected [16]. This phenomenon has been studied in various contexts such as parton energy loss [17, photon [18, 19, 20] and gluon [21] production in a quark-gluon plasma (for a general discussion see [22]). Recently [20], we presented a new approach how to consistently include soft gauge interactions in the computation of a thermal particle production rate. It is formulated in a way that is largely independent of the type of particles whose production we want to study, and can therefore easily be adapted to the case at hand.

The paper is organized as follows. In Sec. 2 we relate the production rate, which describes out-of-equilibrium physics, to a real-time correlation function in equilibrium. The latter can be calculated in thermal field theory, which is subject to the following sections. We describe the physics of collinear emission and outline the relevant momentum scales in Sects. 3.1 and 3.2. In Sec. 3.3 we give a short and qualitative summary how we proceed to obtain the leading order production rate due to collinear emission processes. The rest of Sec. 3 provides all the technical details that are needed to arrive at the final results, and the reader who is not interested in the details of their derivation may skip directly to Eq. (39). In Sec. 4 we present numerical results and we conclude in Sec. 5. The appendix finally explains how to obtain the numerical solutions.

\section{Production rate and thermal field theory}

At lowest order in their Yukawa couplings the production rate of Majorana neutrinos with 4 -momentum $k$ can be written as 4

$$
\frac{\mathrm{d} \Gamma}{\mathrm{d}^{3} k}=\frac{1}{(2 \pi)^{3} k^{0}} f_{\mathrm{F}}\left(k^{0}\right) \operatorname{Tr}\left[k \operatorname{Im} \Sigma_{\mathrm{ret}}(k)\right] .
$$

Here $\Sigma_{\text {ret }}(k)=\Sigma\left(k^{0}+i 0^{+}, \boldsymbol{k}\right)$ is the retarded self-energy for the Majorana neutrinos, and $f_{\mathrm{F}}$ is the Fermi-Dirac distribution.

\footnotetext{
${ }^{4}$ Our formula for the production rate of a spin $1 / 2$-fermion is consistent with those shown in the literature (e.g. [23]) although the overall sign appears to be different. However, with our conventions the self-energy corresponds to $(-1)$ times the Feynman diagrams.
} 


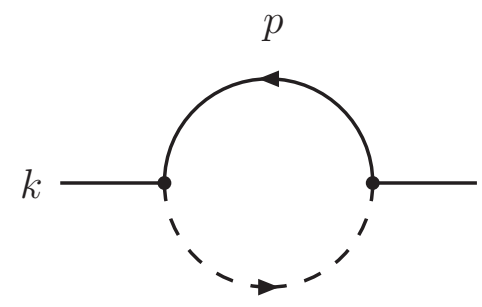

Figure 1: The imaginary part of this diagram gives the production rate due to decay and inverse decay processes. This diagram also determines the inhomogeneous term in Eq. (23).

Due to the Majorana-nature of $N$ there are two types of diagrams contributing to the self-energy which differ by the orientation of the internal fermion lines. We neglect Standard Model CP-violation. Then both types of diagrams give the same contribution. Therefore we consider only one of them, and multiply by 2 to obtain the correct rate. We do this by considering only the self-energy for right-handed $N$ 's, so that the non-vanishing components of the self-energy fit into a $2 \times 2$ matrix which we denote by $\Sigma_{\mathrm{R}}$. Then the rate can be written as

$$
\frac{\mathrm{d} \Gamma}{\mathrm{d}^{3} k}=\frac{2}{(2 \pi)^{3} k^{0}} f_{\mathrm{F}}\left(k^{0}\right) \operatorname{Tr}\left[\bar{\sigma} \cdot k \operatorname{Im} \Sigma_{\mathrm{R}, \mathrm{ret}}(k)\right]
$$

where $\bar{\sigma}^{0}=\mathbb{1}, \bar{\sigma}=-\boldsymbol{\sigma}$.

\section{Production by collinear emission}

\subsection{Production processes}

Without any SM interactions the imaginary part of the self-energy is obtained by cutting the diagram in Fig. 1, with the lines in the loop being tree-level propagators. The corresponding production mechanism is the inverse decay of the massive Majorana neutrino, $\varphi \ell \rightarrow N$.

The typical momentum of a particle in the hot plasma is $O(T)$. We refer to these momenta as 'hard'. In the high-temperature regime, when $M_{N} \lesssim g T$, one cannot neglect the modification of the dispersion relation of SM particles, which is caused by the interactions with other particles in the hot plasma. The dispersion relation for hard particles can be written as $p^{2}=m^{2}$, where $m$ is the so-called asymptotic mass [24]. 5

\footnotetext{
${ }^{5}$ It is oftentimes referred to as $m_{\infty}$.
} 

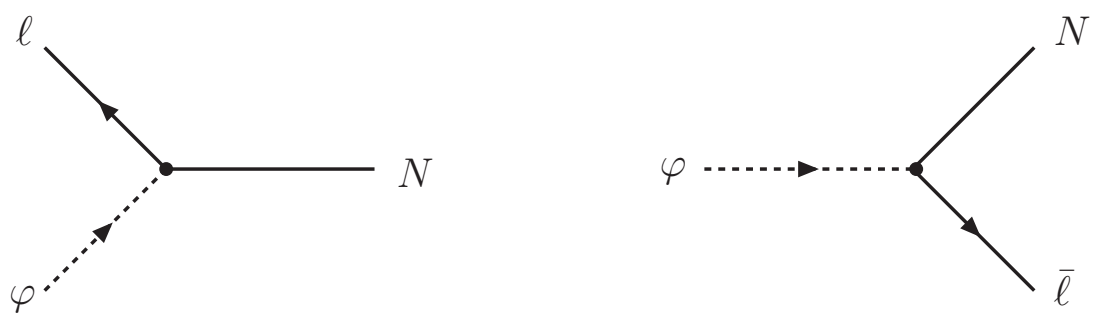

Figure 2: Decay and inverse decay processes that contribute to the production of Majorana neutrinos.

It is given by the self-energy of a hard particle with (nearly) light-like momentum, and the dominant contribution is due to hard loop momenta. Its values for the Higgs and the lepton doublets are given by 6

$$
\begin{aligned}
& m_{\varphi}^{2}=\frac{1}{16}\left(3 g^{2}+g^{\prime 2}+4 h_{t}^{2}+8 \lambda\right) T^{2}, \\
& m_{\ell}^{2}=\frac{1}{16}\left(3 g^{2}+g^{\prime 2}\right) T^{2} .
\end{aligned}
$$

Note that the gauge field contributions to the asymptotic masses for Higgs and leptons are equal (cf. Refs. [25]). However, the Higgs also receives important contributions from the Yukawa interaction with the top quark and from the Higgs self-interaction, so that $m_{\varphi}>m_{\ell}$. All other contributions can be neglected due to the smallness of the corresponding coupling constants. Furthermore, the thermal mass of the Majorana neutrinos can be neglected, so that $M_{N}$ has a temperature-independent value. Therefore, the Higgs and the leptons become "heavier" than the Majorana neutrinos at high $T$.

When $m_{\varphi}+m_{\ell}>M_{N}$ the inverse decay is kinematically forbidden. At even higher temperature, when $m_{\varphi}-m_{\ell}>M_{N}$, the phase space for the decay $\varphi \rightarrow \bar{\ell} N$ of the Higgs boson opens up (see Fig. 2). The decay due to thermal masses has been considered as a mechanism for Majorana neutrino production previously [8, 9]. 7]

When $M_{N} \lesssim g T$, the characteristic feature of both processes described above is that the momenta of the particles are nearly collinear because the relevant masses are small.

\footnotetext{
${ }^{6}$ For scalars the asymptotic mass is the same as the thermal self-energy computed for vanishing momentum, which enters the finite temperature effective potential for the scalar field. It also equals the frequency of scalar field oscillations with zero $\boldsymbol{p}$. For fermions, however, the asymptotic mass is larger than the oscillation frequency for vanishing $\boldsymbol{p}$ by a factor $\sqrt{2} 24$.

${ }^{7}$ In Refs. [8, 9] the oscillation frequency for vanishing $\boldsymbol{p}$ was used instead of the asymptotic mass for fermions.
} 

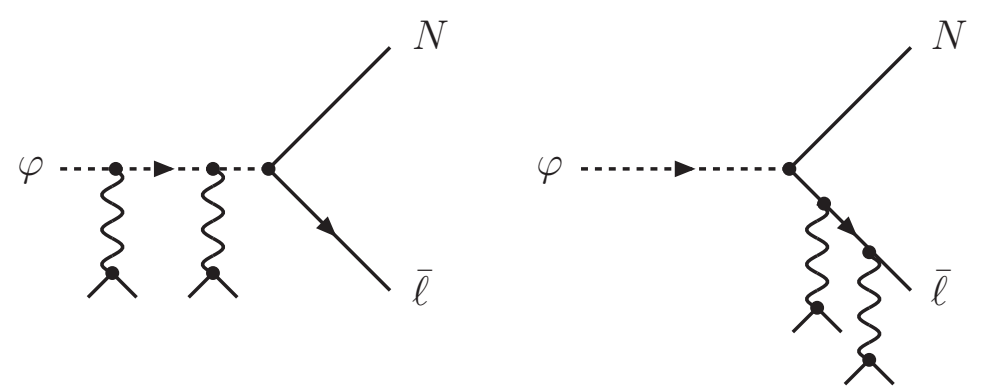

Figure 3: Example for two processes whose interference needs to be taken into account in a consistent leading order treatment.

The angles between the particle momenta are of order $g$. We will show that multiple scattering mediated by soft gauge bosons contributes already at leading order, similar to the thermal production of on-shell photons [18, 19]. The additional couplings are compensated by nearly on-shell propagators. The lepton and the Higgs boson undergo an arbitrary number of scatterings off soft gauge bosons during the 'emission' of the Majorana neutrino which still feels the presence of its 'source'. In order to take this phenomenon into account, one has to resum an infinite set of diagrams, like in [19, 20] for the production of photons from a quark-gluon plasma. This will be dealt with in the rest of Sec. 3 .

\subsection{Momentum scales}

For the processes that we consider the 4-momenta of the emitting and of the emitted particles are almost collinear and close to the light-cone. The 3-momenta point in approximately the same direction, which we represent by the 3 -vector $\boldsymbol{v}$ with $\boldsymbol{v}^{2}=1$. The components parallel to $\boldsymbol{v}$ are denoted by

$$
p_{\|} \equiv \boldsymbol{p} \cdot \boldsymbol{v}
$$

We further define the light-like vector $v \equiv(1, \boldsymbol{v})$.

One has to account for three distinct momentum scales:

1. The emitting particles and the emitted particle both have $p_{\|} \sim T$, which is our hard scale.

2. All 3-momenta perpendicular to $\boldsymbol{v}$ are soft, $\boldsymbol{p}_{\perp} \sim g T$. Furthermore, all momentum components of the exchanged gauge bosons are soft, $q_{\mu} \sim g T$.

3. Finally, all 4-momenta $k$ satisfy $v \cdot k=\left(k_{0}-k_{\|}\right) \sim g^{2} T$.

The hard loop momenta have $k^{2} \sim g^{2} T^{2}$. Therefore the propagators are sensitive to the asymptotic mass $m \sim g T$. 


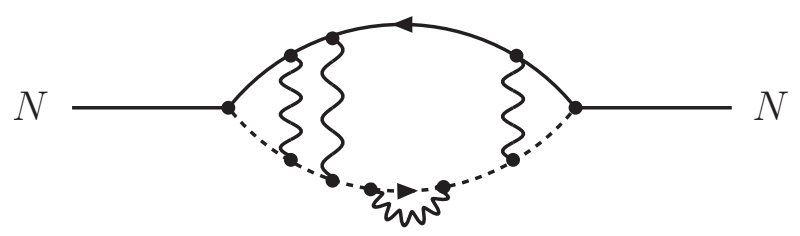

Figure 4: Example for a self-energy diagram whose imaginary part contributes to the leading order production rate.

\subsection{Strategy of the calculation}

We now turn to computing the production rate due the processes discussed in Sec. 3.1. They involve interference terms with an arbitrary number of soft $\mathrm{SU}(2)$ and $\mathrm{U}(1)$ gauge bosons and the corresponding self-energy needed for (33) is given by the sum of all ladder diagrams of the type shown in Fig. 4. We use the approach of Ref. [20] to obtain an integral equation for the self-energy that we can subsequently solve numerically and get the contribution to the production rate. Before going into the details, let us recall the strategy of the calculation [20]:

1. Integrate out the hard field modes. This generates the asymptotic masses for hard particles near the light-cone, i.e. with $p^{2} \sim g^{2} T^{2}$. Furthermore, one obtains the Hard Thermal Loop (HTL) effective action for soft fields, in particular the gauge fields. At leading order no thermal width for the hard particles is generated in this step. The width due to hard-hard interaction is of order $g^{4} T$ and can be neglected. The leading order width is due to soft gauge interactions and enters only at a later stage.

2. Consider 1-loop diagrams with 2 external Majorana neutrinos and with an arbitrary number of external soft gauge bosons, in the limit that the loop momentum and the hard external momenta are nearly collinear. Expand the propagators and vertices in the coupling and keep only the leading order terms. First compute the 2-point function without any gauge boson explicitly. Then set up a recursion relation that relates a given $n$-point function to $(n-1)$-point functions where one of the gauge bosons has been removed. This recursion relation is most easily formulated in terms of a 'current' which is induced by background Majorana neutrino and gauge fields.

3. Finally, integrate out the soft gauge boson background. The gauge bosons then only appear in self-energy insertions (thereby generating the thermal width for 
the lepton and the Higgs boson) and as rungs in the ladder diagrams (see Fig. 41). 8 The new current satisfies an integral equation that is straightforwardly obtained from the one found in step 2. By stripping off the external field we obtain an integral equation for the desired self-energy.

In the remainder of Sec. 3, we will provide all the relevant details to arrive at the final result (39) for the production rate of Majorana neutrinos, following the strategy outlined above.

\subsection{2-point function}

In this section we explicitly compute the 2-point function of the Majorana neutrinos shown in Fig. 1, ignoring any interactions, except that we use the asymptotic masses. It turns out that all $n$-point functions with additional $(n-2)$ soft gauge bosons can be obtained from $(n-1)$-point functions through a simple recursion relation. All simplifications of propagators and vertices which are needed for the general case already appear in the calculation of the 2-point function.

Let us first have a look at the propagators. Keeping only the leading order $g^{2} T^{2}$ terms in the denominator, the scalar propagator with mass $m_{\varphi}$ can be written as [20]

$$
\Delta(p) \equiv \frac{-1}{p^{2}-m_{\varphi}^{2}} \simeq \frac{D_{\varphi}(p)}{2 p_{\|}}
$$

with

$$
D_{a}(p) \equiv \frac{-1}{v \cdot p-\left(\boldsymbol{p}_{\perp}^{2}+m_{a}^{2}\right) /\left(2 p_{\|}\right)} .
$$

Now consider a spin 1/2 propagator. We consider only left-handed fermions propagating in the loop. Thus we can work with 2-component Weyl spinors. One can approximate

$$
S(p) \simeq \frac{D_{\ell}(p)}{2 p_{\|}} \sigma \cdot \widetilde{p}
$$

with

$$
\widetilde{p} \equiv p-\frac{m_{\ell}^{2}}{2 p_{\|}} u,
$$

\footnotetext{
${ }^{8}$ At leading order, only diagrams of the form shown in Fig. 4 are generated. No diagrams with crossed ladder rungs or nested loops occur [19, 20].
} 
where $u \equiv(1,0)$ is the 4 -velocity of the plasma. The matrix $\sigma \cdot \widetilde{p}$ contains terms of order $T, g T$ and $g^{2} T$; higher order terms have been neglected. It is necessary to keep the $g^{2} T$ terms because they give a leading order contribution to the rate in Eq. (3).

If the fermion momentum is on-shell, $p^{2}=m_{\ell}^{2}$, then $\tilde{p}$ is light-like up to higher order terms which we have neglected. Therefore, if we evaluate the loop integral in the imaginary-time formalism and take the imaginary part, the fermion propagator is on-shell, and we can treat $\widetilde{p}$ as a light-like 4 -vector. This allows us to write

$$
\sigma \cdot \widetilde{p}=2 p_{\|} \eta(\widetilde{p}) \eta^{\dagger}(\widetilde{p})
$$

with a 2-component Weyl-spinor $\eta(\widetilde{p})$. Then the fermion propagator becomes

$$
S(p)=\eta(\widetilde{p}) \eta^{\dagger}(\widetilde{p}) D_{\ell}(p)
$$

We choose the 3-axis in the direction of $\boldsymbol{v}$ and expand $\eta(\widetilde{p})$ in powers of $g$,

$$
\eta=\eta_{0}+\eta_{1}+O\left(g^{2}\right)
$$

with

$$
\eta_{0}=\left(\begin{array}{c}
0 \\
1
\end{array}\right), \quad \eta_{1}=-\frac{\boldsymbol{\sigma} \cdot \boldsymbol{p}_{\perp}}{2 p_{\|}} \eta_{0} .
$$

The lower component is $O(1)$ and the upper component is $O(g)$. Since we only need the leading order for each component of $\eta$, which is at most of order $g$, we can write $\eta(p)$ instead of $\eta(\widetilde{p})$ because the difference of $p$ and $\widetilde{p}$ is of order $g^{2} T$.

We use the partial fractioning

$$
D_{\ell}(p) D_{\varphi}(p-k)=\frac{1}{\epsilon(k, \boldsymbol{p})}\left[D_{\ell}(p)-D_{\varphi}(p-k)\right]
$$

where

$$
\epsilon(k, \boldsymbol{p}) \equiv v \cdot k+\frac{\left(\boldsymbol{p}_{\perp}-\boldsymbol{k}_{\perp}\right)^{2}+m_{\varphi}^{2}}{2\left(p_{\|}-k_{\|}\right)}-\frac{\boldsymbol{p}_{\perp}^{2}+m_{\ell}^{2}}{2 p_{\|}}
$$

is the difference of the energy poles of the two propagators. Then it is straightforward to compute the self-energy. It will turn out to be convenient to write it in the form

$$
\Sigma_{\mathrm{R}}(k)=|h|^{2} \int \frac{\mathrm{d}^{3} p}{(2 \pi)^{3}} \eta(p) \widehat{\Sigma}(k, \boldsymbol{p}) .
$$




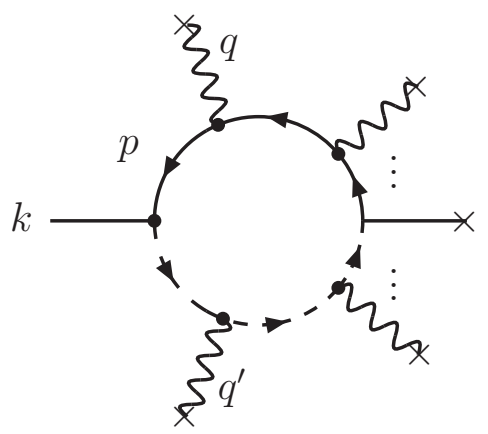

Figure 5: 1-loop diagrams with soft external gauge field lines. Only the 2-point function Fig. 1 needs to be calculated explicitly. The $n$-point functions with $n>2$ are related to the $(n-1)$-point functions by a recursion relation.

It is proportional to $|h|^{2} \equiv \sum_{j}\left|h_{1 j}\right|^{2}$ where the sum goes over the families of leptons. The gauge interactions which will be included are the same for each lepton family, so that this factor can always be extracted. Our result for the 'reduced self-energy' $\widehat{\Sigma}(k, \boldsymbol{p})$ is

$$
\widehat{\Sigma}(k, \boldsymbol{p})=-\frac{d(r) \mathscr{F}\left(k_{\|}, p_{\|}\right)}{2 \epsilon(k, \boldsymbol{p})} \frac{\eta^{\dagger}(p)}{p_{\|}-k_{\|}},
$$

where $d(r)=2$ denotes the dimension of the gauge group representation for lepton and Higgs, and

$$
\mathscr{F}\left(k_{\|}, p_{\|}\right) \equiv f_{\mathrm{F}}\left(p_{\|}\right)+f_{\mathrm{B}}\left(p_{\|}-k_{\|}\right)
$$

From Eq. (18) one then obtains the rate due to the decay of the Higgs or from the inverse decay of the Majorana neutrino, with the diagrams shown in Fig. 2, Explicit results can be found in Sec. 3.8 .

\subsection{Self-energy in soft gauge field background}

Now we consider diagrams with 2 external $N$ 's and, in addition, $(n-2)$ external gauge field lines. These diagrams can be recursively related to diagrams with $(n-3)$ gauge fields. To obtain this relation we pick out one vertex, the leftmost one in Fig. 5, with incoming momentum $k$. As for the 2-point function we can use the approximations (8) and (12) for the propagators. After the partial fractioning (15) this diagram is proportional to the difference of the diagrams in which either the external line with momentum $q$ or the one with momentum $q^{\prime}$ is omitted.

It is convenient to work with the generating functional of the diagrams rather than with the diagrams themselves. We attach background fields to the external lines, 
except to the one on the left. That means that we consider the first derivative of the generating functional. It can be interpreted as a 'current' which is generated by the presence of the background fields.

Here we simplify the treatment of Ref. [20], where first diagrams were considered and then the generating functional was built from them. Instead we start directly with the current. We define $\widehat{J}(k, \boldsymbol{p})$ as the sum of all diagrams in the background of external Majorana neutrino $N$ and of gauge fields $A_{\mu}^{A}$, but without the integral over the 3-momentum $\boldsymbol{p}$, without the trace over the $\mathrm{SU}(2)$ indices, and without the vertex factor $\eta(p)$ (cf. Eq. (17)). Each lepton family gives the same contribution. Therefore we can also pull out the factor $|h|^{2}$ as in Eq. (17). The current without gauge fields, which corresponds to our result from Sec. 3.4, is

$$
\operatorname{tr} \widehat{J}(k, \boldsymbol{p})=\widehat{\Sigma}(k, \boldsymbol{p}) N(k)
$$

Here 'tr' refers to the trace over $\mathrm{SU}(2)$ indices.

For computing higher $n$-point functions it is furthermore convenient to associate the factor $\left(2 p_{\|}\right)^{-1}$ in the scalar propagator $\Delta(p)$ (see Eq. (77) ) with the vertex in the direction of $p$, rather than with the propagator itself. Then the vertex of two Higgs lines with one gauge field with soft momentum $q$ is

$$
\frac{1}{2 p_{\|}}(2 p-q)^{\mu}=v^{\mu}+O(g) .
$$

On the fermion lines we associate the spinors $\eta(p)$ and $\eta^{\dagger}(p)$ not with the propagator, but with the vertices to which the propagator is attached. For a fermion-gauge field vertex one therefore obtains a factor

$$
\eta^{\dagger}(p-q) \bar{\sigma}^{\mu} \eta(p)=v^{\mu}+O(g)
$$

From Eqs. (21) and (22) we only need the leading order contributions, which are equal.

For the two propagators which are connected to the left vertex in Fig. 5 we use the same approximations (8) and (12) as for the computation of the 2-point function, and we use the partial fractioning (15). The resulting two terms are then proportional to diagrams in which either the propagator with momentum $p$ or the one with momentum $p-k$ has been omitted from Fig. 5. Thus, if one also leaves out the vertex factors and the background gauge fields connected to these propagators, each of the two terms gives a $(n-1)$-point function. For the second term in Eq. (15) we obtain a contribution in which the propagator with momentum $p$ has been omitted. We perform a shift in 
the summation variable, $p^{0} \rightarrow p^{0}+q^{0}$. Then the remaining propagators are the same which appear in the $(n-1)$-point function, but with the loop 3-momentum $\boldsymbol{p}$ replaced by $\boldsymbol{p}-\boldsymbol{q}$. Therefore we can write $\widehat{J}(k, \boldsymbol{p})$ in terms of the difference of $\widehat{J}$ times a gauge field and of a gauge field times $\widehat{J}$,

$$
\begin{aligned}
\epsilon(k, \boldsymbol{p}) \widehat{J}(k, \boldsymbol{p})= & -\frac{1}{2} \mathscr{F}\left(k_{\|}, p_{\|}\right) \frac{\eta^{\dagger}(p)}{p_{\|}-k_{\|}} N(k) \\
& +\int_{q}[\widehat{J}(k-q, \boldsymbol{p}) v \cdot A(q)-v \cdot A(q) \widehat{J}(k-q, \boldsymbol{p}-\boldsymbol{q})] .
\end{aligned}
$$

In the imaginary-time formalism

$$
\int_{q} \equiv T \sum_{q^{0}=i \omega} \int \frac{\mathrm{d}^{3} q}{(2 \pi)^{3}}
$$

where $\omega$ is a bosonic Matsubara frequency. Furthermore, $A_{\mu} \equiv A_{\mu}^{A} T^{A}$ contains both $\mathrm{SU}(2)$ and $\mathrm{U}(1)$ gauge fields. Note that we have included the gauge couplings in $A_{\mu}^{A}$. They will appear in the gauge field propagators. Eq. (23) is of the same form as Eq. (21) in [20], only the inhomogeneous term on the RHS is different.

\subsection{Integrating out the soft gauge bosons}

Now we use Eq. (23) to integrate out the soft gauge fields. This will give us the $N$ self-energy including soft gauge interactions. To see how it works, we write Eq. (23) schematically as $\widehat{J}=N+A \widehat{J}$, leaving out all terms and all factors which are not essential for this purpose. We iterate this equation once, which gives $\widehat{J}=N+A(N+$ $A \widehat{J})$. Now we integrate this expression over the gauge fields. The term linear in $A$ drops out and we obtain $\langle\widehat{J}\rangle=N+\langle A A \widehat{J}\rangle$, where $\langle\cdots\rangle$ denotes the path integral over the soft $A$-field. In [20] we have shown that at leading order $\langle A A \widehat{J}\rangle=\langle A A\rangle\langle\widehat{J}\rangle$. In this way we obtain a closed equation for $\langle\widehat{J}\rangle$.

Now we can become more explicit. We iterate Eq. (23) once and we take the trace over the $\mathrm{SU}(2)$ indices. This allows us to bring all terms in the same order,

$$
\begin{aligned}
& \epsilon(k, \boldsymbol{p}) \operatorname{tr}\langle\widehat{J}(k, \boldsymbol{p})\rangle=-\frac{d(r)}{2} \mathscr{F}\left(k_{\|}, p_{\|}\right) \frac{\eta^{\dagger}(p)}{p_{\|}-k_{\|}} N(k) \\
& \quad+2 \int_{q} \int_{q^{\prime}} \frac{1}{v \cdot(k-q)} \operatorname{tr}\left[\left\langle v \cdot A(q) v \cdot A\left(q^{\prime}\right)\right\rangle\left\langle\widehat{J}(k, \boldsymbol{p})-\widehat{J}\left(k, p_{\|}, \boldsymbol{p}_{\perp}-\boldsymbol{q}_{\perp}\right)\right\rangle\right] .
\end{aligned}
$$


Inside the integral we have approximated $\epsilon(k-q, \boldsymbol{p}) \simeq \epsilon(k-q, \boldsymbol{p}-\boldsymbol{q}) \simeq v \cdot(k-q)$, i.e. we have neglected the terms containing transverse momenta and thermal masses, even though they are of the same order as the term we kept. This is possible because the gauge field propagator separately depends on $q_{0} \sim g T$ and $q_{\|} \sim g T$, and not on their difference $v \cdot q \sim g^{2} T$. Therefore the terms we have omitted only contribute to a higher order shift of the integration variable $q_{\|}$.

For the $\mathrm{SU}(2) \times \mathrm{U}(1)$ gauge fields one has at leading order

$$
\left\langle A_{\mu}(q) A_{\nu}\left(q^{\prime}\right)\right\rangle=\tilde{\delta}\left(q+q^{\prime}\right)\left[C_{2}(r) g^{2} \Delta_{\mu \nu}(q)+y_{\ell}^{2} g^{\prime 2} \Delta_{\mu \nu}^{\prime}(q)\right]
$$

where in the imaginary-time formalism

$$
\tilde{\delta}\left(q+q^{\prime}\right) \equiv T^{-1} \delta_{q_{0}+q_{0}^{\prime}, 0}(2 \pi)^{3} \delta\left(\boldsymbol{q}+\boldsymbol{q}^{\prime}\right)
$$

Furthermore, $C_{2}(r)$ is the quadratic Casimir operator which for the fundamental representation of $\mathrm{SU}(2)$ equals $3 / 4$, and $y_{\ell}=-1 / 2$ is the lepton hypercharge. Finally, $\Delta_{\mu \nu}$ and $\Delta_{\mu \nu}^{\prime}$ are the HTL resummed propagators of the $\mathrm{SU}(2)$ and $\mathrm{U}(1)$ gauge fields.

On the RHS of Eq. (25) the unknown function $\widehat{J}$ does not depend on $q_{0}$ or $q_{\|}$. Therefore these integrals can be performed. One encounters the expression

$$
I\left(k, \boldsymbol{q}_{\perp}\right) \equiv T \sum_{q^{0}=i \omega} \int \frac{\mathrm{d} q_{\|}}{2 \pi} \frac{v^{\mu} v^{\nu} \Delta_{\mu \nu}(q)}{v \cdot(k-q)},
$$

and similarly with $\Delta_{\mu \nu}^{\prime}$. Here $k^{0}$ is purely imaginary, $k^{0}=i \tilde{\omega}$, where $\tilde{\omega}$ is a fermionic Matsubara frequency. After performing the sum over the bosonic Matsubara frequencies $\omega$, one has to analytically continue to $k^{0}+i 0^{+}$, where $k^{0}$ is now real. Using standard results for the HTL resummed propagators [26] and the sum rule of Ref. [27] one obtains

$$
I\left(k^{0} \pm i 0^{+}, \boldsymbol{k}, \boldsymbol{q}_{\perp}\right) \simeq \mp \frac{i}{2} T\left(\frac{1}{\boldsymbol{q}_{\perp}^{2}}-\frac{1}{\boldsymbol{q}_{\perp}^{2}+m_{\mathrm{D}}^{2}}\right)
$$

where $m_{\mathrm{D}}$ is a Debye mass. In the SM the Debye masses of the $\mathrm{SU}(2)$ and $\mathrm{U}(1)$ gauge bosons $m_{\mathrm{D}}$ and $m_{\mathrm{D}}^{\prime}$ are given by [28]

$$
m_{\mathrm{D}}^{2}=\frac{11}{6} g^{2} T^{2}, \quad m_{\mathrm{D}}^{\prime 2}=\frac{11}{6} g^{\prime 2} T^{2} .
$$

The RHS of Eq. (29) no longer depends on $k$. The only $k$-dependence of $I$ is through the sign of the imaginary part of the frequency. 
Now we strip off the background $N$-field from $\widehat{J}$ which gives $\widehat{\Sigma}$ (see Eq. (20)). The equation for $\widehat{\Sigma}(k, \boldsymbol{p})$ then reads

$$
\begin{aligned}
i \epsilon(k, \boldsymbol{p}) \widehat{\Sigma}(k, \boldsymbol{p})= & -\frac{i}{2} d(r) \mathscr{F}\left(k_{\|}, p_{\|}\right) \frac{\eta^{\dagger}(p)}{p_{\|}-k_{\|}} \\
& +\int \frac{\mathrm{d}^{2} q_{\perp}}{(2 \pi)^{2}} \mathscr{C}\left(\boldsymbol{q}_{\perp}\right)\left[\widehat{\Sigma}(k, \boldsymbol{p})-\widehat{\Sigma}\left(k, p_{\|}, \boldsymbol{p}_{\perp}-\boldsymbol{q}_{\perp}\right)\right]
\end{aligned}
$$

with the kernel

$$
\mathscr{C}\left(\boldsymbol{q}_{\perp}\right) \equiv T\left[C_{2}(r) g^{2}\left(\frac{1}{\boldsymbol{q}_{\perp}^{2}}-\frac{1}{\boldsymbol{q}_{\perp}^{2}+m_{\mathrm{D}}^{2}}\right)+y_{\ell}^{2} g^{\prime 2}\left(\frac{1}{\boldsymbol{q}_{\perp}^{2}}-\frac{1}{\boldsymbol{q}_{\perp}^{2}+m_{\mathrm{D}}^{\prime 2}}\right)\right] .
$$

The integral equation (31) is of the same form as the one for the production of photons [20], only the inhomogeneous term is different. Both $\epsilon(k, \boldsymbol{p}) \sim g^{2} T$ and $\mathrm{d}^{2} q_{\perp} \mathscr{C}\left(\boldsymbol{q}_{\perp}\right) \sim g^{2} T$. Therefore the integral term which resums the multiple interactions with an arbitrary number of soft gauge bosons is of the same order as the term which corresponds to the tree-level (inverse) decay.

\subsection{Production rate of Majorana neutrinos}

To compute the trace over spinor indices in Eq. (44) we expand

$$
\bar{\sigma} \cdot k=2 k_{\|} \chi_{0} \chi_{0}^{\dagger}+\frac{M_{N}^{2}}{2 k_{\|}} \eta_{0} \eta_{0}^{\dagger}+O\left(g^{3} T\right)
$$

where $\eta_{0}$ is given by Eq. (14), and

$$
\chi_{0}=\left(\begin{array}{l}
1 \\
0
\end{array}\right)
$$

Since the first term in Eq. (33) is $O(T)$ and the second is $O\left(g^{2} T\right)$, both terms in Eq. (13), and consequently both components of $\widehat{\Sigma}(k, \boldsymbol{p})$, contribute to the leading order production rate.

Now, if one has the solutions to the equations

$$
\begin{aligned}
& i \epsilon(k, \boldsymbol{p}) \boldsymbol{f}\left(\boldsymbol{p}_{\perp}\right)-\int \frac{\mathrm{d}^{2} q_{\perp}}{(2 \pi)^{2}} \mathscr{C}\left(\boldsymbol{q}_{\perp}\right)\left[\boldsymbol{f}\left(\boldsymbol{p}_{\perp}\right)-\boldsymbol{f}\left(\boldsymbol{p}_{\perp}-\boldsymbol{q}_{\perp}\right)\right]=2 \boldsymbol{p}_{\perp}, \\
& i \epsilon(k, \boldsymbol{p}) \psi\left(\boldsymbol{p}_{\perp}\right)-\int \frac{\mathrm{d}^{2} q_{\perp}}{(2 \pi)^{2}} \mathscr{C}\left(\boldsymbol{q}_{\perp}\right)\left[\psi\left(\boldsymbol{p}_{\perp}\right)-\psi\left(\boldsymbol{p}_{\perp}-\boldsymbol{q}_{\perp}\right)\right]=1,
\end{aligned}
$$


which were obtained in [19, 30] for transverse and (virtual) longitudinal photon production, then the solution to Eq. (31) is given by

$$
\widehat{\Sigma}(k, \boldsymbol{p})=-\frac{i}{2} \frac{d(r) \mathscr{F}\left(k_{\|}, p_{\|}\right)}{p_{\|}-k_{\|}}\left(\begin{array}{c}
-\left(f^{1}+i f^{2}\right) / p_{\|} \\
\psi
\end{array}\right) .
$$

Unless one ignores the interactions with soft gauge fields by putting $\mathscr{C}$ equal to zero, the imaginary part of $k^{0}$ is no longer relevant in Eqs. (35) and (36). Only its sign has entered by determining the sign of $\mathscr{C}$ through Eq. (29).

We may now choose $\boldsymbol{v}$ in the direction of $\boldsymbol{k}$, so that $\boldsymbol{k}_{\perp}=\mathbf{0}$, and $k_{\|}=|\boldsymbol{k}| \equiv k$. Combining Eqs. (44), (14), (17), (33), (34), (37) and using

$$
f_{\mathrm{F}}(k)\left[f_{\mathrm{F}}(p)+f_{\mathrm{B}}(p-k)\right]=-f_{\mathrm{F}}(p) f_{\mathrm{B}}(k-p)
$$

we obtain for the production rate

$$
\frac{\mathrm{d} \Gamma}{\mathrm{d}^{3} k}=-\frac{d(r)|h|^{2}}{(2 \pi)^{3} 2 k} \int \frac{\mathrm{d}^{3} p}{(2 \pi)^{3}} \frac{1}{k-p_{\|}} f_{\mathrm{F}}\left(p_{\|}\right) f_{\mathrm{B}}\left(k-p_{\|}\right) \operatorname{Re}\left[\frac{k}{2 p_{\|}^{2}} \boldsymbol{p}_{\perp} \cdot \boldsymbol{f}+\frac{M_{N}^{2}}{k} \psi\right] .
$$

The integral equations (35) and (36) for $\boldsymbol{f}$ and $\psi$ can only be solved numerically which is greatly simplified by transforming them via a Fourier transformation into a boundary-value problem for a differential equation as shown in appendix A.

The Yukawa interaction couples Majorana neutrinos to SM leptons with opposite chirality. In the massless limit where helicity equals chirality the collinear emission is forbidden by angular momentum conservation. This argument is not affected by the thermal masses for fermions because they do not violate chiral symmetry. The contribution containing $f$ corresponds to helicity changing processes, and therefore it vanishes when $\boldsymbol{p}_{\perp}$ is zero. $\psi$ does not vanish in the collinear limit and corresponds to helicity conserving processes. Therefore $\psi$ vanishes in the limit $M_{N} \rightarrow 0$.

\subsection{Tree-level (inverse) decay}

In addition to solving the full integral equations (35) and (36), we want to explicitly calculate the contribution from the tree-level processes shown in Fig. 22 in order to study how strongly the multiple soft scattering affects the production rate. This corresponds to neglecting the integral term in (31) completely, and the equation becomes a purely algebraic one. In this case one has to keep a small imaginary part of $k^{0}$ in $\epsilon(k, \boldsymbol{p})$. 
Taking the real part of the trace in (39) and performing the integration over $\boldsymbol{p}_{\perp}$ yields

$$
\frac{\mathrm{d} \Gamma^{\text {tree }}}{\mathrm{d}^{3} k}=\frac{d(r)|h|^{2}}{4(2 \pi)^{3} k^{2}} \int_{p_{-}}^{p_{+}} \frac{\mathrm{d} p_{\|}}{2 \pi} \frac{1}{\left|p_{\|}\right|} f_{\mathrm{F}}\left(p_{\|}\right) f_{\mathrm{B}}\left(k-p_{\|}\right)\left[p_{\|}\left(M_{N}^{2}-m_{\varphi}^{2}\right)-\left(k-p_{\|}\right) m_{\ell}^{2}\right] .
$$

The integration limits are determined by the conditions that the energy of the lepton be positive and that the particles be on-shell, which means that $\epsilon(k, \boldsymbol{p})=0$ has a real solution for $\left|\boldsymbol{p}_{\perp}\right|$. For the two processes shown in Fig. 2, this gives the integration limits

$$
p_{ \pm}=\frac{X \pm \sqrt{Y}}{2 M_{N}^{2}}
$$

where

$$
\begin{aligned}
X & \equiv M_{N}^{2}+m_{\ell}^{2}-m_{\varphi}^{2}, \\
Y & \equiv\left(m_{\varphi}+m_{\ell}+M_{N}\right)\left(m_{\varphi}-m_{\ell}+M_{N}\right)\left(m_{\varphi}+m_{\ell}-M_{N}\right)\left(m_{\varphi}-m_{\ell}-M_{N}\right) .
\end{aligned}
$$

It is easy to see that $Y \geq 0$ only if either $m_{\varphi} \geq M_{N}+m_{\ell}$ (which corresponds to the decay of the Higgs boson) or $M_{N} \geq m_{\varphi}+m_{\ell}$ (which corresponds to the inverse decay of the Majorana neutrino). For $m_{\varphi}-m_{\ell}<M_{N}<m_{\varphi}+m_{\ell}$ we therefore obtain $\mathrm{d} \Gamma^{\text {tree }} / \mathrm{d}^{3} k=0$.

\section{Numerical results}

To obtain numerical results we have to specify several parameters. The mass $M_{N}$ of the Majorana neutrino and its Yukawa couplings are unconstrained by low-energy neutrino physics. We have chosen the exemplary value $M_{N}=10^{7} \mathrm{GeV}$ and we always

plot our rates divided by $|h|^{2}=\sum_{j}\left|h_{1 j}\right|^{2}$. The SM couplings are evaluated at the scale $\mu=2 \pi T$ using the 1-loop renormalization group equations [29]. To determine the Higgs self-coupling $\lambda$ we have assumed $m_{H}=150 \mathrm{GeV}$ for the zero temperature Higgs mass.

First consider the integrated production rate $\Gamma=\int \mathrm{d}^{3} k\left(\mathrm{~d} \Gamma / \mathrm{d}^{3} k\right)$ as a function of $z=M_{N} / T$. Our results are shown in Fig. 6. At small $z$ the 'tree-level rate' (40) is due to the decay of Higgs bosons, while at large $z$ it results from the inverse decay of heavy Majorana neutrinos. At intermediate $z$ none of these processes is kinematically allowed, and the rate vanishes. It is remarkable that the full line which includes soft 


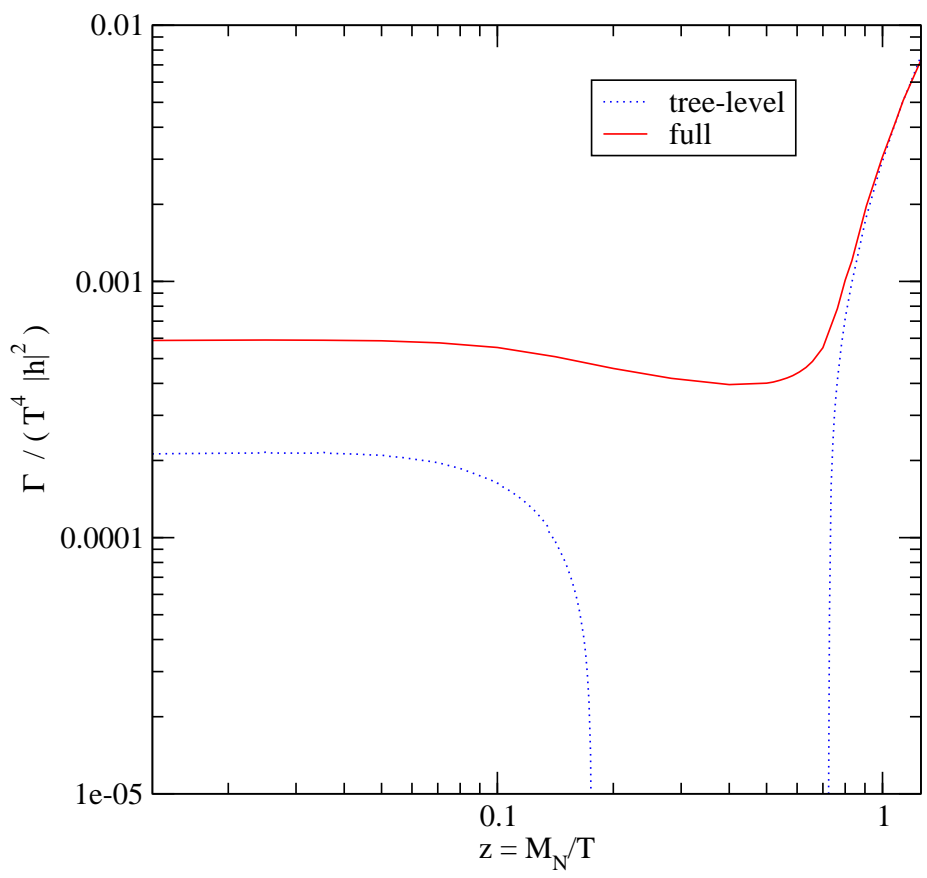

Figure 6: Number of produced Majorana neutrinos per unit time and unit volume as a function of $z \equiv M_{N} / T$. The dotted curve is the result without any soft gauge interactions. The full line includes an arbitrary number of soft gauge interactions.

gauge interactions is very smooth in the regions where the decay of the Higgs boson becomes kinematically forbidden.

One can see that the full rate is larger than the tree-level rate by about a factor 3 at small $z$. It decreases only mildly when the tree-level processes are forbidden. When the inverse decay process sets in, the difference between tree-level rate and the complete rate goes to zero. This is expected, since the collinear enhancement is a relativistic effect and it disappears when the Majorana neutrinos become non-relativistic. One should emphasize that the strong enhancement caused by the soft gauge interactions does not signal a breakdown of perturbation theory, because all contributions discussed above are leading order.

It is also interesting to consider the contribution due to helicity changing and helicity conserving processes separately (cf. the discussion at the end of Sec. 3.7). The rate of helicity changing processes does not vanish in the limit $M_{N} \rightarrow 0$, and should therefore be dominant at small $z$. The results are shown in Fig. 7 . We clearly see that the helicity changing process dominates at high temperatures and the helicity conserving 


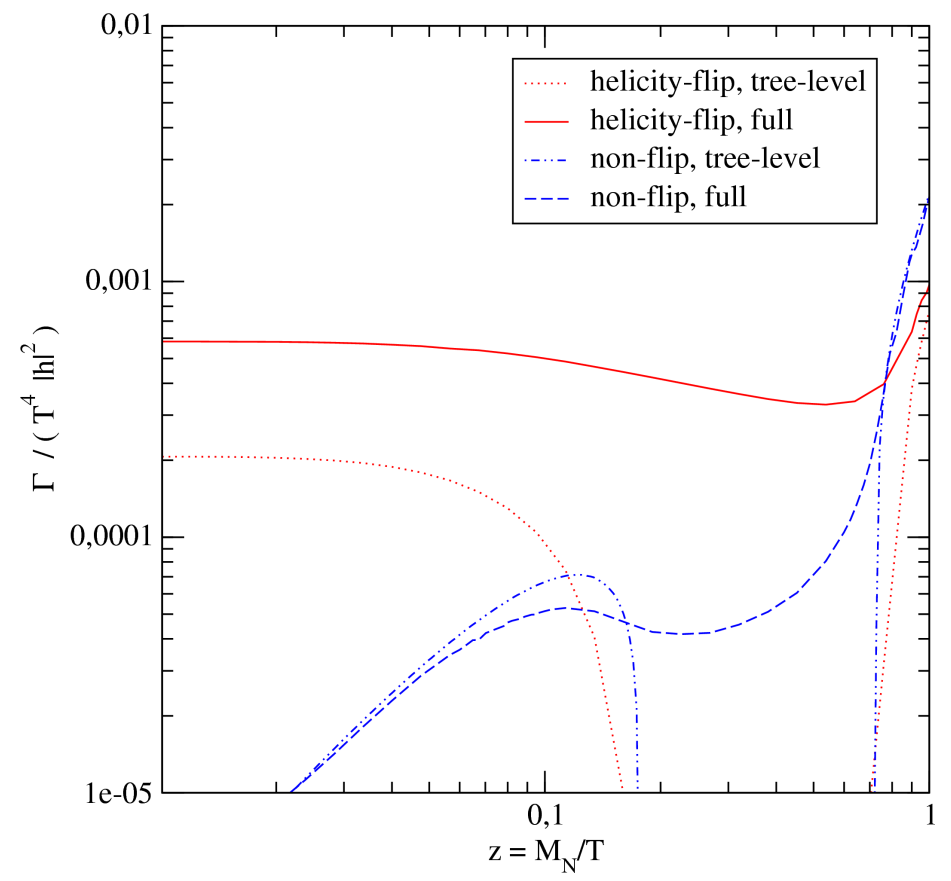

Figure 7: Contributions to the production rate of Majorana neutrinos due to helicity changing and helicity conserving processes as a function of $z \equiv M_{N} / T$.

process is relevant only at low temperatures, $T \sim M_{N}$, while it becomes negligible at $T \gg M_{N}$. This is true both for the tree-level processes and the processes which include multiple soft scattering.

Finally we study the momentum spectrum of the produced heavy neutrinos. If it was thermal, the rate would be proportional to the Fermi-Dirac distribution $f_{\mathrm{F}}(k)$. In Fig. 8 we show the ratio of the differential production rate and $f_{\mathrm{F}}(k)$ for two different temperatures. For $z=0.1$ the production occurs both via a tree-level process (in this case, the decay of the Higgs boson) and via processes involving multiple soft scattering whereas for $z=0.5$ the tree-level processes are kinematically forbidden. We see that the spectrum is peaked in the infrared which means that the typical momenta of the produced neutrinos are smaller than in equilibrium. This holds both for helicity changing and for helicity conserving processes.

\section{$5 \quad$ Summary and Conclusions}

In this paper we have studied the production of relativistic Majorana neutrinos, relevant for models of thermal leptogenesis, in a hot electroweak plasma. Based on our 


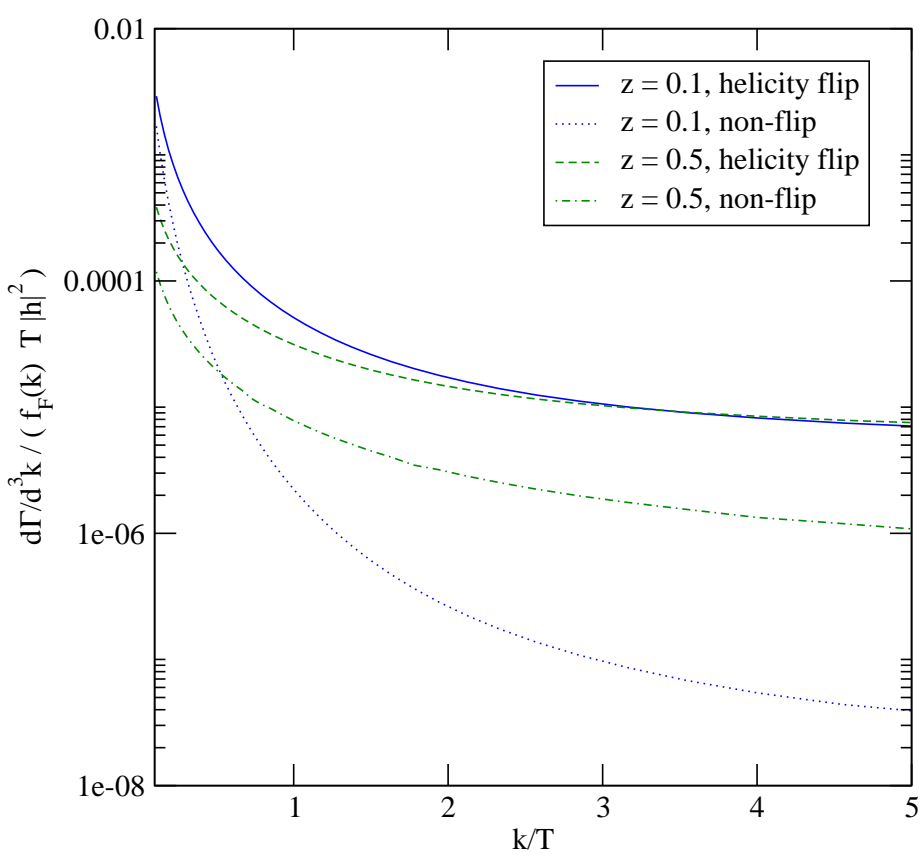

Figure 8: Differential production rate divided by Fermi-Dirac distribution function for two different temperatures.

previous work [20], we have obtained an equation which sums all leading order collinear production processes. It includes the tree-level processes-the decay of the Higgs boson as well as the inverse decay of the Majorana neutrino-and in addition processes involving multiple scattering mediated by soft gauge boson exchange. All these turn out to contribute at leading order in the coupling constants and have never been included in previous treatments of thermal leptogenesis.

Numerically, we find a very pronounced increase in the thermal production rate when soft gauge interactions are included. At high temperatures, when the production via tree-level Higgs decay is allowed, the rate increases by about a factor 3 . When the tree-level processes are forbidden, the rate in units of temperature drops only slightly, and the production of Majorana neutrinos remains effective. When the temperature is close to $M_{N}$ the rate is not significantly affected by soft gauge interactions. We further showed that the production rate is dominated by helicity changing processes and that helicity conserving (inverse) decays only play a significant role at low temperatures, $T \sim M_{N}$. In addition to the production rate, we have studied the momentum spectrum of the produced Majorana neutrinos and found that it is strongly peaked in the infrared. 
The spectrum is thus not even approximately thermal.

The leading order production rate of heavy Majorana neutrinos also receives contributions from $2 \leftrightarrow 2$ scattering processes. They have been considered previously in the context of thermal leptogenesis (see e.g. [8, 12, 13, 14, 15]), but a complete and consistent leading order calculation has not been done so far. In [8] the Higgs decay was found to dominate over the $2 \leftrightarrow 2$ processes at high temperature. In this paper we have shown that adding soft gauge interactions to the Higgs decay process leads to a strong enhancement of the production rate. Therefore it would be very interesting to see how the processes discussed here, together with the $2 \leftrightarrow 2$ scattering processes affect various scenarios of leptogenesis.

Acknowledgments This work was supported in part through the DFG funded Graduate School GRK 881.

\section{A Solving the integral equations}

The $\boldsymbol{p}_{\perp}$-integrals of the functions $\boldsymbol{f}$ and $\psi$ in Eq. (39) can be obtained without solving the integral equations (35) and (36). This is achieved by Fourier transformation which turns Eq. (35) and (36) into boundary value problems. Our approach which is described in this Appendix closely follows Refs. [27, 30].

\section{A.1 From integral equation to differential equation}

Eqs. (35) and (36) are of the same form as the integral equation for the production of transverse and of longitudinal photons. Following [27, 30] we Fourier transform 9

$$
\boldsymbol{f}(\boldsymbol{b})=\int \mathrm{d}^{2} b e^{i \boldsymbol{p}_{\perp} \cdot \boldsymbol{b}} \boldsymbol{f}\left(\boldsymbol{p}_{\perp}\right), \quad \psi(\boldsymbol{b})=\int \mathrm{d}^{2} b e^{i \boldsymbol{p}_{\perp} \cdot \boldsymbol{b}} \psi\left(\boldsymbol{p}_{\perp}\right) .
$$

Then the integral over the perpendicular components in (39) can be replaced by the limit of their Fourier transforms for $b \rightarrow 0$ :

$$
\begin{aligned}
\int \frac{\mathrm{d}^{2} p_{\perp}}{(2 \pi)^{2}} \operatorname{Re}\left[\boldsymbol{p}_{\perp} \cdot \boldsymbol{f}\left(\boldsymbol{p}_{\perp}\right)\right] & =\lim _{b \rightarrow 0} \operatorname{Im} \boldsymbol{\nabla} \cdot \boldsymbol{f}(\boldsymbol{b}), \\
\int \frac{\mathrm{d}^{2} p_{\perp}}{(2 \pi)^{2}} \operatorname{Re} \psi\left(\boldsymbol{p}_{\perp}\right) & =\lim _{b \rightarrow 0} \operatorname{Re} \psi(\boldsymbol{b})
\end{aligned}
$$

\footnotetext{
${ }^{9}$ We use the same symbol for the functions $\boldsymbol{f}, \psi$ and their Fourier transforms.
} 
The integral equations (35), (36) now turn into differential equations for $\boldsymbol{f}(\boldsymbol{b})$ and $\psi(\boldsymbol{b})$. With $\boldsymbol{k}_{\perp}=\mathbf{0}$ and $k^{2}=M_{N}^{2}$ we can write

$$
\epsilon(k, \boldsymbol{p})=\beta\left(\boldsymbol{p}_{\perp}^{2}+M_{\mathrm{eff}}^{2}\right)
$$

where

$$
\beta=\frac{k_{\|}}{2 p_{\|}\left(p_{\|}-k_{\|}\right)}, \quad M_{\mathrm{eff}}^{2}=\frac{p_{\|}\left(p_{\|}-k_{\|}\right) M_{N}^{2}-k_{\|}\left(p_{\|}-k_{\|}\right) m_{\ell}^{2}+k_{\|} p_{\|} m_{\varphi}^{2}}{k_{\|}^{2}} 1 .
$$

Then we obtain differential equations which closely resemble those in [30],

$$
\begin{aligned}
& -i \beta\left(\Delta-M_{\mathrm{eff}}^{2}\right) \boldsymbol{f}(\boldsymbol{b})-\mathscr{K}(b) \boldsymbol{f}(\boldsymbol{b})=-i 2 \nabla \delta(\boldsymbol{b}), \\
& -i \beta\left(\Delta-M_{\mathrm{eff}}^{2}\right) \psi(\boldsymbol{b})-\mathscr{K}(b) \psi(\boldsymbol{b})=\delta(\boldsymbol{b})
\end{aligned}
$$

where $b \equiv|\boldsymbol{b}|$, and

$$
\mathscr{K}(b)=T\left[C_{2}(r) g^{2} D\left(m_{\mathrm{D}} b\right)+y_{\ell}^{2} g^{\prime 2} D\left(m_{\mathrm{D}}^{\prime} b\right)\right]
$$

with [27, 30]

$$
D(x)=\frac{1}{2 \pi}\left[\gamma_{\mathrm{E}}+\ln \frac{x}{2}+K_{0}(x)\right] .
$$

Here, $\gamma_{\mathrm{E}}=0.5772 \ldots$ denotes the Euler-Mascheroni constant and $K_{0}(x)$ is a modified Bessel function.

The convergence of the Fourier integrals enforces the boundary conditions

$$
\lim _{b \rightarrow \infty} \boldsymbol{f}(\boldsymbol{b})=0, \quad \lim _{b \rightarrow \infty} \psi(\boldsymbol{b})=0
$$

In the limit $b \rightarrow 0$ all terms which contain no derivatives can be neglected in (A.6), (A.7), and we obtain the limiting behaviour

$$
\begin{aligned}
& \boldsymbol{f}(\boldsymbol{b})=c_{f} \frac{\boldsymbol{b}}{b^{2}}+O(b), \\
& \psi(\boldsymbol{b})=c_{\psi} \ln b+O\left(b^{0}\right) .
\end{aligned}
$$

The constants $c_{f}, c_{\psi}$ are fixed by the $\delta$-functions in Eqs. (A.6), (A.7) which gives

$$
\begin{aligned}
c_{f} & =\frac{1}{\pi \beta}, \\
c_{\psi} & =\frac{i}{2 \pi \beta} .
\end{aligned}
$$


Due to rotational invariance we must have $\boldsymbol{f}(\boldsymbol{b})=h(b) \boldsymbol{b}$ and $\psi(\boldsymbol{b})=\psi(b)$. In terms of the new function $h(b)$ the RHS of (A.2) becomes $2 \lim _{b \rightarrow 0} \operatorname{Im} h(b)$. We numerically solve the ODEs for $h(b)$ and $\psi(b)$

$$
\begin{aligned}
& -i \beta\left(\partial_{b}^{2}+\frac{3}{b} \partial_{b}-M_{\text {eff }}^{2}\right) h(b)-\mathscr{K}(b) h(b)=0 \\
& -i \beta\left(\partial_{b}^{2}+\frac{1}{b} \partial_{b}-M_{\text {eff }}^{2}\right) \psi(b)-\mathscr{K}(b) \psi(b)=0
\end{aligned}
$$

for $b>0$. The conditions (A.10), (A.13) and (A.14) are then sufficient to determine the solutions unambiguously.

\section{A.2 Numerical procedure}

Finally we want to describe our algorithm that we used to obtain the solutions shown in Sec. 4, Both equations (A.16) and A.15) can be solved by the same method and we only describe the procedure in terms of (A.16) explicitly.

For the numerical solution, it proves convenient to split the function into a tree-level part and another part coming from multiple soft scattering,

$$
\psi(b)=\psi_{0}(b)+\psi_{1}(b) .
$$

The function $\psi_{0}$ solves (A.7) with $D(b) \equiv 0$ and can be written in terms of Bessel functions. For $b \rightarrow 0$, the function $D(b)$ behaves like $D(b) \sim b^{2} \ln b$. This implies that the limiting behaviour of $\psi_{0}$ for $b \rightarrow 0$ is also given by Eq. (A.14). Therefore $\psi_{1}(b)$ must be regular at $b=0$. The general solution for $\psi_{1}$ can be written as

$$
\psi_{1}(b)=c_{1} \psi_{1}^{(1)}(b)+c_{2} \psi_{1}^{(2)}(b)+\psi_{1}^{(\text {part })}(b)
$$

where $\psi_{1}^{\text {(part) }}$ is a particular solution of the inhomogeneous equation while the linearly independent solutions $\psi_{1}^{(i)}$ solve the corresponding homogeneous equation. One can choose $\psi_{1}^{(1)}$ such that it has the limiting behavior of Eq. (A.12), and a $\psi_{1}^{(2)}$ which is regular for $b \rightarrow 0$. Thus we must have $c_{1}=0$. The other constant is then found by imposing (A.10) and we obtain

$$
c_{2}=-\lim _{b \rightarrow \infty} \frac{\psi_{1}^{(\text {part })}(b)}{\psi_{1}^{(2)}(b)} .
$$

Numerically, one has to choose finite values for ' $b \rightarrow 0$ ' and ' $b \rightarrow \infty$ '. We have found $b_{0}=10^{-5} T^{-1}$ and $b_{\infty}=30 T^{-1}$ to be a reasonable choice, giving stable numerical solutions.

The algorithm providing us with the desired solution is then the following: 
1. Solve the homogeneous ODE for $\psi_{1}(b)$ with $\psi_{1}\left(b_{0}\right)=1, \psi_{1}^{\prime}\left(b_{0}\right)=0$ to obtain $\psi_{1}^{(2)}(b)$

2. Solve the inhomogeneous ODE for $\psi_{1}(b)$ with $\psi_{1}\left(b_{0}\right)=i, \psi_{1}^{\prime}\left(b_{0}\right)=0$ to obtain $\psi_{1}^{(\text {part })}(b)$

3. Compute $c_{2}$ via (A.19). Then, due to the initial conditions chosen in the previous steps, the desired result is given by $\operatorname{Re} \psi_{1}(b \rightarrow 0)=\operatorname{Re} c_{2}$.

In order to find $\operatorname{Im} h_{1}(b \rightarrow 0)$ we can use the same algorithm, only in the second step it is more convenient to choose $h_{1}\left(b_{0}\right)=1, h_{1}^{\prime}\left(b_{0}\right)=0$ because then $\operatorname{Im} h_{1}(b \rightarrow 0)=\operatorname{Im} c_{2}$.

\section{References}

[1] E. Komatsu et al., Five-Year Wilkinson Microwave Anisotropy Probe (WMAP) Observations: Cosmological Interpretation, Astrophys. J. Suppl. 180 (2009) 330. arXiv:0803.0547 [astro-ph]]

[2] A. D. Sakharov, Violation of CP invariance, C asymmetry, and baryon asymmetry of the Universe, Pisma Zh. Eksp. Teor. Fiz. 5 (1967) 32.

[3] For reviews see e.g. A. D. Dolgov, Baryogenesis, 30 Years after, Lectures given at the 25th ITEP Winter School arXiv:hep-ph/9707419]; A. Riotto, Theories of Baryogenesis, Lectures delivered at the Summer School in High Energy Physics and Cosmology, Trieste, Italy, 29 June - 11 July 1998 arXiv:hep-ph/9807454; M. Dine and A. Kusenko, The origin of the matter-antimatter asymmetry, Rev. Mod. Phys. 76 (2004) 1 arXiv:hep-ph/0303065]; W. Buchmüller, Baryogenesis40 years after, arXiv:0710.5857v2].

[4] M. Fukugita and T. Yanagida, Baryogenesis without grand unification, Phys. Lett. B 174 (1986) 45.

[5] V. Kuzmin, V. Rubakov and M. Shaposhnikov, On anomalous electroweak baryonnumber nonconservation in the early universe, Phys. Lett. B 155 (1985) 36.

[6] P. Minkowski, $\mu \rightarrow$ er at a rate of one out of $10^{9}$ muon decays?, Phys. Lett. B 67 (1977) 421; M. Gell-Mann, P. Ramond and R. Slansky, Complex spinors and unified theories, in: Proc.Supergravity Stony Brook Workshop, New York 1979, ed. 
by P. van Nieuwenhuizen and D.Z. Freedman, North-Holland Publ. Co., Amsterdam, 1979; T. Yanagida, Horizontal symmetries and masses of neutrinos, Prog. Theor. Phys. 64 (1980), 1103

[7] L. Covi, N. Rius, E. Roulet and F. Vissani, Finite temperature effects on CP violating asymmetries, Phys. Rev. D 57 (1998) 93 arXiv:hep-ph/9704366|; M. Garny, A. Hohenegger and A. Kartavtsev, Medium corrections to the CP-violating parameter in leptogenesis, Phys. Rev. D 81 (2010) 085028. [arXiv:1002.0331 [hep-ph]]

[8] G. F. Giudice, A. Notari, M. Raidal, A. Riotto and A. Strumia, Towards a complete theory of thermal leptogenesis in the SM and MSSM, Nucl. Phys. B 685 (2004) 89 arXiv:hep-ph/0310123.

[9] C. P. Kiessig, M. Plümacher and M. H. Thoma, Decay of a Yukawa fermion at finite temperature and applications to leptogenesis, arXiv:1003.3016v2 [hep-ph]].

[10] W. Buchmüller and S. Fredenhagen, Quantum mechanics of baryogenesis, Phys. Lett. B 483 (2000) 217 arXiv:hep-ph/0004145]; A. De Simone and A. Riotto, Quantum Boltzmann Equations and Leptogenesis, JCAP 0708 (2007) 002 arXiv:hep-ph/0703175]; M. Beneke, B. Garbrecht, M. Herranen and P. Schwaller, Finite Number Density Corrections to Leptogenesis, Nucl. Phys. B 838 (2010) 1 arXiv:1002.1326 [hep-ph]]; M. Beneke, B. Garbrecht, C. Fidler, M. Herranen and P. Schwaller, Flavoured Leptogenesis in the CTP Formalism, Nucl. Phys. B 843 (2010) 177 arXiv:1007.4783 [hep-ph]]; B. Garbrecht, Leptogenesis: The Other Cuts, arXiv:1011.3122 [hep-ph]].

[11] A. Anisimov, W. Buchmüller, M. Drewes and S. Mendizabal, Leptogenesis from Quantum Interference in a Thermal Bath, Phys. Rev. Lett. 104 (2010) 121102 arXiv:1001.3856 [hep-ph]].

[12] A. Pilaftsis and T. E. J. Underwood, Resonant leptogenesis, Nucl. Phys. B 692 (2004) 303 arXiv:hep-ph/0309342].

[13] W. Buchmüller, P. Di Bari and M. Plumacher, Leptogenesis for pedestrians, Annals Phys. 315, 305 (2005) arXiv:hep-ph/0401240.

[14] S. Davidson, E. Nardi and Y. Nir, Leptogenesis, Phys. Rept. 466 (2008) 105 arXiv:0802.2962 [hep-ph]]. 
[15] F. Hahn-Woernle, M. Plumacher and Y. Y. Y. Wong, Full Boltzmann equations for leptogenesis including scattering, JCAP 0908 (2009) 028 [arXiv:0907.0205 [hep$\mathrm{ph}]$.

[16] L. D. Landau and I. Pomeranchuk, Limits of applicability of the theory of bremsstrahlung electrons and pair production at high-energies, Dokl. Akad. Nauk Ser. Fiz. 92 (1953) 535; also printed in Collected Papers of L.D. Landau, Edited by D. Ter Haar, Pergamon Press, 1965; A. B. Migdal, Bremsstrahlung And Pair Production In Condensed Media At High-Energies, Phys. Rev. 103 (1956) 1811.

[17] R. Baier, Y. L. Dokshitzer, A. H. Mueller, S. Peigne and D. Schiff, Radiative energy loss and $p($ T)-broadening of high energy partons in nuclei, Nucl. Phys. B 484 (1997) 265 arXiv:hep-ph/9608322.

[18] P. Aurenche, F. Gelis and H. Zaraket, Landau-Pomeranchuk-Migdal effect in thermal field theory, Phys. Rev. D 62 (2000) 096012 [arXiv:hep-ph/0003326].

[19] P. Arnold, G. D. Moore and L. G. Yaffe, Photon Emission from Ultrarelativistic Plasmas, JHEP 0111 (2001) 057 arXiv:hep-ph/0109064; Photon emission from quark gluon plasma: Complete leading order results, JHEP 0112 (2001) 009 arXiv:hep-ph/0111107.

[20] D. Besak and D. Bödeker, Hard Thermal Loops for soft or collinear external momenta, JHEP 1005 (2010) 007 arXiv:1002.0022 [hep-ph]].

[21] P. Arnold, G. D. Moore and L. G. Yaffe, Photon and gluon emission in relativistic plasmas, JHEP 0206 (2002) 030 arXiv:hep-ph/0204343.

[22] P. B. Arnold, G. D. Moore and L. G. Yaffe, Effective kinetic theory for high temperature gauge theories, JHEP 0301 (2003) 030 arXiv:hep-ph/0209353].

[23] M. Bolz, A. Brandenburg and W. Buchmüller, Thermal production of gravitinos, Nucl. Phys. B 606 (2001) 518 arXiv:hep-ph/0012052; J. Pradler, Electroweak Contributions to Thermal Gravitino Production [arXiv: 0708.2786v1 [hep-ph]].

[24] H.A. Weldon, Effective fermion masses of order gT in high-temperature gauge theories with exact chiral invariance, Phys. Rev. D 26 (1982) 2789. 
[25] V. V. Lebedev and A. V. Smilga, Supersymmetric sound, Nucl. Phys. B 318 (1989) 669; S. Caron-Huot, On supersymmetry at finite temperature, Phys. Rev. D 79 (2009) 125002 [arXiv:0808.0155 [hep-th]].

[26] E. Braaten and R. D. Pisarski, Soft Amplitudes in Hot Gauge Theories: A General Analysis, Nucl. Phys. B 337 (1990) 569; J. Frenkel and J. C. Taylor, High Temperature Limit of Thermal QCD, Nucl. Phys. B 334 (1990) 199.

[27] P. Aurenche, F. Gelis and H. Zaraket, A simple sum rule for the thermal gluon spectral function and applications, JHEP 0205 (2002) 043 [arXiv:hep-ph/0204146].

[28] M. E. Carrington, Effective potential at finite temperature in the Standard Model, Phys. Rev. D 45 (1992) 2933.

[29] H. Arason et al., Renormalization-group study of the standard model and its extensions: The standard model, Phys. Rev. D 46 (1992) 3945; B. Schrempp and M. Wimmer, Top quark and Higgs boson masses: Interplay between infrared and ultraviolet physics, Prog. Part. Nucl. Phys. 37, 1, 1996 arXiv:hep-ph/9606386].

[30] P. Aurenche, F. Gelis, G.D. Moore and H. Zaraket, Landau-PomeranchukMigdal resummation for dilepton production, JHEP 0212 (2002) 006 arXiv:hep-ph/0211036]. 